ARTIGO

\title{
EL CONOCIMIENTO COMO PROBLEMA EN LA UNIVERSIDAD DEL SIGLO XXI
}

\author{
O CONHECIMENTO COMO PROBLEMA EM UMA UNIVERSIDADE DO SÉCULO
}

XXI

\section{KNOWLEDGE AS A PROBLEM IN THE 21ST CENTURY UNIVERSITY}

\author{
José M. Bautista-Vallejo \\ Universidad de Huelva - España \\ Rafael M. Hernández-Carrera \\ Universidad Internacional de la Rioja - España \\ Rodrigo Matos de-Souza \\ Universidade de Brasília - Brasil
}

\begin{abstract}
Resumen: Tradicionalmente, la misión desempeñada por la universidad tenía que ver con la cultura, la ciencia y la profesión. En la actualidad, nuevas formas de producción y gestión del conocimiento, dentro y fuera de la universidad, plantean en el marco de esas tres funciones, como mínimo, el hecho de que estamos ante la necesidad de una nueva sociedad del conocimiento. La universidad tiene la misión de descubrir, y también difundir, nuevos conocimientos. La sociedad, a su vez, precisa de soluciones permanentes a sus inacabables problemas. Cómo se relacionan ambos aspectos tensionantes en el presente y en el futuro es una gran incógnita. Acontecimientos como la pandemia por COVID-19 no han hecho más reforzar esta discusión. Este trabajo tiene el objetivo de reunir información actualizada sobre los significados de conocimiento en el ámbito universitario e identificar los aspectos relevantes y la controversia que puede haber sobre la cuestión. Para ello se llevó a cabo una revisión documental descriptiva, con un análisis a través de una serie de categorías. Se manejaron más de 300 documentos de entre los que fueron seleccionados 47. Entre los resultados destacamos el hecho de las importantes incoherencias y controversias que diversos tipos de conocimiento generan en el seno universitario, algo que afecta a los currículos formativos y la forma en que la universidad se relaciona con el conocimiento y con el mundo.
\end{abstract}

Palabras clave: Universidad; conocimiento; currículum; enseñanza-aprendizaje.

Resumo: Tradicionalmente, a missão realizada pela universidade tinha a ver com a cultura, a ciência e a profissionalidade. Hoje, novas formas de produção e gestão do conhecimento, dentro e fora da universidade, evidenciam o fato de que estamos enfrentando a necessidade de uma nova sociedade do conhecimento, no mínimo, dentro da estrutura dessas três funções. A universidade tem a missão de descobrir e também disseminar novos conhecimentos. A sociedade, por sua vez, precisa de soluções permanentes para seus problemas sem fim. Como estas duas tensões estão relacionadas no presente e no futuro é uma grande incógnita. Eventos como a pandemia da COVID-19 apenas reforçaram esta discussão. Este artigo tem como objetivo reunir informações atualizadas sobre os significados do futuro 
é uma grande incógnita. Eventos como a pandemia da COVID-19 apenas reforçaram esta discussão. Este artigo tem como objetivo reunir informações atualizadas sobre os significados do conhecimento no ambiente universitário e identificar os aspectos relevantes, bem como as controvérsias que possam existir sobre o assunto. Para este fim, foi realizada uma revisão documental descritiva, com uma análise através de uma série de categorias. Mais de 300 documentos foram tratados, dos quais 47 foram selecionados. Entre os resultados destacamos o fato das importantes inconsistências e controvérsias que vários tipos de conhecimento geram dentro da universidade, algo que afeta os currículos de treinamento e a forma como a universidade se relaciona com o conhecimento e com o mundo.

Palavras-chave: Universidade; conhecimento; currículo; ensino-aprendizagem.

\begin{abstract}
Traditionally, the mission carried out by the university had to do with culture, science and the profession. Today, new forms of knowledge production and management, both inside and outside the university, raise within the framework of these three functions, at least, the fact that we are facing the need for a new knowledge society. The university has the mission of discovering, and also disseminating, new knowledge. Society, in turn, needs permanent solutions to its endless problems. How these two tense aspects relate in the present and in the future is a great unknown. Events such as the COVID-19 pandemic have only reinforced this discussion. This paper aims to gather up-to-date information on the meanings of knowledge in the university environment and to identify relevant aspects and controversy that may exist on the issue. To this end, a descriptive documentary review was carried out, with an analysis through a series of categories. More than 300 documents were handled, from which 47 were selected. Among the results we highlight the fact that there are important inconsistencies and controversies that various types of knowledge generate within the university, something that affects the training curricula and the way in which the university relates to knowledge and the world.
\end{abstract}

Keywords: University; knowledge; curriculum; teaching-learning.

\title{
Introducción
}

El papel que juega el nuevo modo de producción de conocimiento y su contenido están haciendo surgir desde la universidad, y desde una parte de la sociedad, algunas de las siguientes preguntas: ¿pueden adaptarse las viejas y las nuevas universidades a las nuevas demandas y condiciones? ¿Qué aspecto tendría una adaptación llevada a cabo con éxito? ¿Qué tipo de intereses han de ser contemplados para que de verdad pueda legitimarse el cambio en la universidad?

Tradicionalmente, la misión desempeñada por la universidad tenía que ver con la cultura, la ciencia y la profesión, manifestada por sus ejes de enseñanza, investigación y extensión. Así pues, con la nueva producción de conocimiento surgen más preguntas: ¿Se trata de un conocimiento como respuesta condicionada a la sociedad? ¿Debe ser una radical orientación hacia los problemas más urgentes del ser humano y de las comunidades? ¿Dónde hay que hacer más hincapié, en la cultura, la ciencia o la profesión?

En la universidad de hoy, problemas tan complejos como el de la apertura total de sus puertas al alumnado, la masificación, la burocratización, la defectuosa relación entre profesores 
y alumnos, la escasa funcionalidad y el desajuste de los espacios y el tiempo de aprendizaje, el desajuste estructural entre la enseñanza superior y el empleo, la escasa investigación y la pobre respuesta desde ésta a los problemas reales de la sociedad, las relaciones entre lo público y lo privado, etc. (BRUNNER, 2014; GUZMÁN-VALENZUELA, 2017), ponen de nuevo de manifiesto que la universidad podría encontrarse en el centro de la discusión sobre los problemas que afectan a la sociedad y cada uno de sus miembros. Acontecimientos como la pandemia por COVID-19 no han hecho más reforzar esta discusión (MATOS-DE-SOUZA, 2020).

Así, cabe especular sobre elementos centrales de los espacios universitarios, como el conocimiento, la enseñanza y el aprendizaje, y los viejos problemas que acarrea la entrada en acción de tales elementos, dado que no faltarán a partir de ahora informes y estudios que afirmen que una parte de la universidad que conocemos debe desaparecer en la era de las Tecnologías de la Información y Comunicación (TIC) (BROWN, BROCK, \& ZÁVODSKÁ, 2019).

Se trata de análisis recurrente. Un ejemplo de ello lo tenemos en uno de los contextos universitarios más dinámicos del mundo, el británico (BECHER, 1989; COMMITTEE OF SCOTTISH UNIVERSITY PRINCIPALS, 1991; SCOTT, 1995; BARNETT; 1997). Visto de otra forma, ya en el nuevo milenio, el Horizon Report: 2017 Higher Education, de Adams, Cummins, Davis, Freeman, Hall, \& Ananthanarayanan (2017), los estudios de Bautista Vallejo y López Jara (2019) sobre temas tan concretos y controvertidos como las competencias o el acuerdo entre las universidades británicas para combatir al COVID-19, el llamado Knowledge Exhange Concordat (2020).

En el caso de Ronald Barnett (1997) sostiene que la estructura profunda del sistema de educación superior de masas concierne ahora a la experiencia del estudiante, donde se ha reducido el contacto entre los profesores y los estudiantes, y además han cambiado las relaciones pedagógicas en el sentido de conferir mayor grado de responsabilidad al propio estudiante. Sin embargo, surge la pregunta, ¿hasta qué punto el establecimiento de estas relaciones basadas en las TIC va a transformar la relación educativa tradicional docenteestudiante?

Esta relación, que dio luz verde a la aparición de las universidades en el siglo XI, aparece cada día más deteriorada por figuras como la creación de conocimiento y la investigación. La universidad tiene la misión de descubrir, y también difundir, nuevos conocimientos. La sociedad, a su vez, precisa de soluciones permanentes a sus inacabables problemas. Cómo se relacionan ambos aspectos tensionantes en el presente y en el futuro es una gran incógnita.

Así pues, el objetivo de este trabajo es doble. Por un lado, reunir información actualizada 
sobre la temática en cuestión, a saber, el impacto de las nuevas relaciones entre los miembros de la comunidad universitaria, en el contexto de una de las funciones de la universidad: la producción de conocimiento. Por otro lado, identificar los aspectos relevantes y la controversia que puede haber sobre la cuestión.

La estrategia seguida ha sido la de llevar a cabo una revisión documental descriptiva (ICART ISERN Y CANELA-SOLER, 1994; KAUARK, MANHÃES Y MEDEIROS, 2010) de una serie de documentos seleccionados entre múltiples fuentes consultadas, en bases de datos y buscadores especializados, fundamentalmente Web of Science (WOS), Dialnet, Google Académico. Estas bases permitieron el acceso a bases consistentes y confiables y el manejo y análisis de un gran volumen de publicaciones científicas a través de una serie de categorías, que tienen las características de ser significativas, claras, excluyentes y replicables (BARDIN, 1996), en una espiral auto-reflexiva continua (HERNÁNDEZ CARRERA, 2014). Las categorías usadas fueron: funciones de la universidad, conocimiento en la universidad, currículum universitario, enseñanza y aprendizaje en la universidad, función docente en la universidad. Se manejaron más de 300 documentos de entre los que fueron seleccionados 41 .

\section{La transformación de la universidad en tiempos de COVID-19}

Tras un siglo sin pandemias, la reciente por COVID-19 ha atrapado a la ciudadanía de todo el mundo. La misma recorre el planeta entero y cuenta en este momento con informes que describen una situación de millones de infectados por el virus y una cifra elevada de muertos (SOHRABI, ALSAFI, O’NEILL, KHAN, KERWAN, AL-JABIR, IOSIFIDIS, \& AGHA, 2020).

En mayor o menor medida, gobiernos del mundo tomaron una serie de medidas que cambiaron la forma de vivir y trabajar de millones de personas. Estas medidas, llevadas a cabo de una forma casi simultánea y espontánea y, en la mayoría de los casos, acelerada, abarcaron todo el espectro social y, lógicamente, a la educación (Muñoz, \& Lluch, 2020), profundizando y acelerando procesos de precarización del trabajo de tal forma que no se conseguiría hacer por voluntad política (STANDING, 2017).

Entre ellas estaba el teletrabajo. El mismo, referido a las instituciones educativas, contribuyó de forma acelerada a la virtualización de la enseñanza-aprendizaje en las instituciones (BOUZA MORA, 2020; WANG, ZHANG, ZHAO, ZHANG, \& JIANG, 2020). Muchas trabajaron sin descanso para no detener su programación docente y otros tantos, mismo con dificultades ante los diferentes recursos tecnológicos, para no perder el empleo. Ello les 
llevó a introducir todo tipo de recomendaciones y normas para el trabajo a distancia y la enseñanza virtual. Para llevar a cabo esta nueva forma de desarrollar la educación se hizo uso de las muchas herramientas que hasta el momento habían sido utilizadas con relativa frecuencia y eficacia. Sin duda, esta nueva forma de trabajar va a tener un impacto en la manera de enseñar y aprender de millones de profesores y estudiantes en todo el mundo. Sin embargo, los resultados son aún una enorme incógnita.

En el ámbito universitario, el caso de las instituciones chinas, lugar de procedencia del primer foco vírico, afectó a millones de miembros, lo cual ha sido muy significativo (WANG, CHENG, YUE, \& MCALEER, 2020). Medidas similares se han sucedido por gran cantidad de países de todo el mundo (SUND, 2020).

En este sentido, algunas de las recomendaciones recientes empezarán a evidenciarse en el periodo postpandemia: una demanda de sistemas educativos más flexibles y accesibles, menos costosos y a los que la persona pueda incorporarse a lo largo de su ciclo vital o vida profesional (GEORGE Y MARETT, 2019) evidenciando lo que algunos empiezan a llamar de uberización del trabajo docente (VENCO, 2019; SILVA, 2019).

En ello, parece que se comienza definitivamente a demandar una educación que no se centre solamente en los sujetos en su etapa escolar, sino en toda la ciudadanía, a través de metodologías adaptadas que abarquen la educación formal, no formal e informal. Estas metodologías exigirán ser no escolarizantes, para aulas que hasta ahora fueron concebidas desde el espíritu racionalista (transmisión, recepción-memorización, repetición), para ser reemplazadas por nuevas funciones educadoras donde los roles de la misma y del profesorado sean transformadores desde la libertad.

\section{La nueva producción del conocimiento}

Al margen de la visión del conocimiento que pueda tenerse, algo que se relaciona con su producción y ámbitos de aplicación, lo que se está dando hoy es un cambio en la manera de producirse el mismo (BARNETT, \& BENGTSEN, 2019). Si no fuera porque una de las instituciones implicadas es la universidad, podría afirmarse que la cuestión no es transcendente del todo. Pero en la universidad el conocimiento no sólo se genera, también se difunde, se transforma para ser enseñado. Es decir, la universidad (en el sentido pretendido en Europa desde el tratado de Bologna y sus áreas de influencia) está obligada a transmitir paquetes de información en un acto docente, con el objetivo de que sean aprendidos (BAUTISTA VALLEJO, 2005). 
Se ha mencionado al aprendizaje. Si se sostiene que el conocimiento está cambiando, habrá que observar, también, la cuestión de hasta qué punto el aprendizaje se está transformando. Pues bien, este nuevo modo de producción del conocimiento funciona dentro de un contexto de aplicación en el que los problemas no se hallan encuadrados en el marco de una estructura disciplinar, sino que es transdisciplinar, antes que mono o multidisciplinar; el mismo se lleva a cabo en formas no jerárquicas, organizadas de forma heterogénea que son esencialmente transitorias y que no están siendo institucionalizados principalmente dentro de las estructuras de la universidad, como ya observaran Gibbons, Limoges, Nowotny, \& Schwartzman (1997).

Si bien esto, aparte de este modo 1 y modo 2 de conocimiento, Barnett (2004) sugiere un modo 3, aquel que supone un proceso de conocimiento activo, en lugar de algo que es externo a los individuos; se trata de algo que aporta sentido e identidad. Es decir, el modo 3 es la base para el currículum y la pedagogía, en el sentido de que el currículum tiene el objetivo de la transformación del ser humano y la pedagogía en sí misma debe caracterizarse por la incertidumbre, con un conocimiento vagamente enmarcado, provisional y abierto. El currículum, entonces, debe ser diseñado para que insista en que los estudiantes se enfrenten y se comprometan con las incertidumbres y dilemas en su campo de conocimiento (WHEELAHAN, 2005).

Si la acción de conocer es algo interno y no externo, entonces tanto currículum como pedagogía deben involucrar a la persona que conoce, es decir, a la que aprende, no sólo como conocedor, también debe hacerlo como persona, en cuya profundidad el conocimiento llevará a cabo su obra transformadora, siempre y cuando los procesos educativos no perturben a la persona que aprende y conoce (DZIB GOODIN, CASTEVICH, HOGAN, SANDERS, SLOVEC, \& YELIZAROV, 2015).

En la práctica, ocurre que estos modos de conocimiento -más familiar, más nuevo y más ontológico- mantienen una relativa convivencia en el contexto de las instituciones universitarias y su formalismo (DE KRUMMEL, BAUTISTA-VALLEJO, HERNÁNDEZ-CARRERA, \& ESPIGARES-PINAZO, 2020), con la excepción del tercero, el más necesario hoy. A pesar de ello, la perspectiva del conocimiento más disciplinar, más familiar, goza de una importancia en términos más o menos absolutos, aunque bajo una concepción comunicativo-transmisiva, no sólo en el seno de la universidad, también la sociedad, y sobre todo ella, a través de todos los agentes que tienen que ver con el conocimiento, trata de dar un lugar en toda su integridad a lo que investigación y conocimiento pueden proveer desde un elevado desarrollo.

Sin embargo, al proponer Barnett $(2004,2019)$ un modo 3 de conocimiento, está 
subrayando una forma de proceder que no cuenta aún con espacios para su desarrollo en las universidades.

Desde finales del siglo XX, la sociedad fue "definida" como "sociedad del conocimiento" $^{1 "}$ (BARNETT, 1997; COMISIÓN DE LAS COMUNIDADES EUROPEAS, 1997; ALFONSO SÁNCHEZ, 2016). El mismo Barnett (2004) advierte sobre el impacto en el mundo de una serie de profundos cambios de naturaleza social, económica, epistémica, tecnológica y cultural, de los cuales pueden extraerse dos puntos generales (BARNETT, 2004).

En primer lugar, la consideración de que el mundo es radicalmente desconocido en condiciones de "super-complejidad". Así, nuestro control sobre el mundo es ahora siempre frágil, lo que implica que hay una importante "brecha epistemológica" entre lo que conocemos del mundo y nuestro conocimiento sobre cómo actuar en el mundo. Dado que la certeza no es posible, debemos actuar incluso ante la incertidumbre. Es decir, nuestros propios sistemas de conocimiento y tecnologías están produciendo un cambio reflexivo, en el cual lo producido se presenta como catalizador de futuros cambios. Es así que este elemento reflexivo está en el nexo entre el conocimiento y el cambio que se produce siempre más rápido, provocando consiguientemente una situación de cambio esencialmente desconocido.

En segundo lugar, la reflexividad construida de nuestros sistemas de conocimiento tiene repercusiones en los sujetos de forma individual. Los crecientes niveles de satisfacción en conceptos, ideas y, por supuesto, discursos manejados, necesitan de individuos si ellos de cualquier forma van a dar sentido al mundo que están encarando. Es por ello que la reflexividad a nivel de cada individuo -es decir, la capacidad crítica de irse interrogando sobre el universo que nos rodea- es una necesidad relacionada con la asimilación y acomodación del nuevo orden, en el que, además, necesitamos desarrollar la confianza y la capacidad para hacerlo posible.

Barnett (2004) se pregunta entonces si el currículum, la pedagogía y la propia institución universitaria están hechas para esta incertidumbre. La respuesta es, de momento, no.

Sin duda, esta es una idea que da visibilidad al problema del conocimiento cuando se sitúa en la universidad. De esto se sigue un conjunto de actitudes y formas de obrar de quienes forman la universidad: profesores-investigadores y alumnado. Más aún, Barnett (2004) sugiere una forma de relacionarse con el mundo, desde dentro de las universidades, en donde son disposiciones básicas el cuidado, la reflexión, la humildad, la capacidad de ser crítico, la

\footnotetext{
${ }^{1}$ Comprendemos que esta definición, por así decir, está enmarcada por una influencia de los debates noventistas sobre inteligencias colectiva, sociedad del conocimiento y por una centralidad del lugar de habla desde los centro económicos del norte global, cuando caminamos para procesos más concretos de vigilancia por la tecnología en las primeras décadas del siglo XXI y con la distancia que podemos tomar hoy, siendo testigos del futuro que muchos de estos autores pensaban distantes, entendemos que su definición tiene algunas limitaciones.
} 
receptividad, la resistencia, el coraje y la quietud. Pero, ¿algunas de éstas son desarrolladas de forma consciente en la universidad? La respuesta es igualmente no.

Este nuevo y necesario modo de conocimiento afecta no sólo a qué conocimiento se produce, sino también a cómo se produce, el contexto en el que se persigue, la forma en que se organiza, el sistema de recompensas que utiliza y los mecanismos que controlan la calidad de aquello que se produce.

Este núcleo y contexto de producción y gestión del conocimiento tiene innegables repercusiones sobre la organización de los procesos de enseñanza y aprendizaje en la universidad (GARCÍA-PEÑALVO, 2016). Por tener en cuenta sólo un dato. Es claro que la universidad trasmite información, pero, ¿la transmite imaginativamente?

Ante la expresión de que la función propia de una universidad es la de la adquisición imaginativa del conocimiento, porque la imaginación es una manera de iluminar los hechos, de sonsacar los principios generales que se aplican a los hechos, lo que posibilita a las personas la construcción de la visión de un nuevo mundo, preserva el entusiasmo de la vida por la sugerencia de propósitos satisfactorios, etc. De ser así, ¿es ésta la dedicación fundamental de la universidad? En opinión de Finn (2018), no.

\section{Obsolescencia del conocimiento y necesidad de innovación}

Una de las cuestiones más interesantes sobre la relación conocimiento y universidad es la relativa a la obsolescencia del conocimiento y su impacto sobre los currícula y programas (SKIBA, 2017). Cabe, incluso, hacerse la pregunta: el conocimiento que se produce en la universidad, ¿sirve para algo?

Entender la situación planteada, cuyo origen es la evolución del conocimiento y de sus modos de producción, desde la propia universidad es una importante necesidad. Las nuevas exigencias planteadas a los sistemas de educación superior se corresponden con los cambios de la estructura de los saberes, de las formas tradicionales de organización, división y especialización del conocimiento, de la circulación y apropiación del mismo, del papel social de las profesiones. Sin embargo, ocurre que todo eso no se asienta de manera segura en los sistemas universitarios, los cuales, por lo general, son conservadores, teóricos, inflexibles y obsoletos. En este sentido, la mayor queja viene por parte del alumnado, durante y tras egresar de la universidad (BAUTISTA-VALLEJO, ESPIGARES-PINAZO, \& HERNÁNDEZCARRERA, 2019).

La situación no solo responde a un cambio de acciones, también supone un cambio de lenguaje. Saber y conocimiento son dos términos que están en el núcleo de esta idea. Entre 
ambos hay diferencias, lo que lleva a algunos autores a tomar posición en relación a cada uno de ellos.

Es conocida la postura de Lyotard (1994). Este opina que el conocimiento es el conjunto de enunciados que denotan o describen los objetos, excluyendo cualquier otro enunciado, con la condición, además, de que son susceptibles de ser declarados verdaderos o falsos. Por su parte, el saber va más allá de esta caracterización. Este hace referencia a una competencia que excede la determinación y la aplicación de criterios de verdad y que se extiende a los criterios de eficacia (cualificación técnica), de justicia y/o felicidad (sabiduría ética), de belleza sonora, cromática (sensibilidad auditiva, visual, etc.). El saber, así concebido, alude a un "conjunto de competencias" y, sobre todo, al sujeto que las realiza, o sea, a la "persona sabio".

Sin embargo, el conocimiento a lo largo de la historia no ha tenido una trayectoria lineal. Esta ha sido interrumpida en múltiples ocasiones, por una diversidad inmensa de acontecimientos que han afectado al ser humano (GIBBONS, LIMOGES, NOWOTNY, \& SCHWARTZMAN, 1997; BURKE, 2015). Como consecuencia, la complicación de las tareas y los problemas sociales a través de la historia de la humanidad fueron demandando saberes diferenciados, profundos y complejos a la vez, lo que ha devuelto para la historia social del conocimiento un panorama de increíbles avances y de futuros inciertos y hasta desconsoladores.

Uno de los efectos de la falta de continuidad fue que se hizo más grande el distanciamiento entre el conocimiento y la persona. En esta relación, el lenguaje se convirtió en el instrumento mediador, la cultura se puso a su servicio y las instituciones que fueron inventadas limitaron la espontaneidad del conocimiento a través de una organización, almacenamiento y especialización del mismo para responder al objetivo de su distribución.

Sin embargo, el resultado final ofrece un enorme contraste en muchas de las instituciones universitarias, donde la realidad desborda lo institucional. En el marco de una economía de alta tecnología y la necesidad de desarrollarla constantemente para sobrevivir en un mercado mundial cada vez más competitivo (BRUNNER, 2014; BONARDI, BRIS, BRÜLHART, DANTHINE, JONDEAU, ROHNER, THOENIG, 2020), la política universitaria no ha entendido hasta qué punto estamos ante la necesidad de una nueva sociedad del conocimiento.

La falta de reconocimiento está llegando a los extremos que Barnett y Bengtsen (2019) definen: la relación entre conocimiento y universidad se ha viciado. Por un lado, el conocimiento se ha separado del mundo y, por extensión, la universidad anima a esta separación entendiendo que el conocimiento característicamente reside en la universidad, con poco contacto con el mundo. 


\section{Currículum y enseñanza-aprendizaje en la universidad}

De entre las funciones de la universidad (enseñanza, investigación y profesión), la de enseñar, que se corresponde con transmitir el conocimiento acumulado durante generaciones es, sin duda, la menos extraño para la universidad tradicional (GARCÍA-PEÑALVO, 2016).

Lo que se produce es, por tanto una serie de incoherencias. Por un lado, hay una incoherencia en las sugerencias, con diversos y conflictivos mensajes que tienen lugar en las instituciones universitarias. Barnett (2004) ya planteaba preguntas como las siguientes: ¿el profesorado va a centrar su tarea sobre los resultados o competencias específicas del trabajo de los estudiantes? ¿Van a centrar este trabajo y su tiempo en proporcionar habilidades genéricas y transferibles dentro del esquema curricular? Estas preguntas se relacionan estrechamente con las estrategias de enseñanza y aprendizaje que se emplean, la cuales son la unión de teorías científicas e implícitas que las sostienen, así como de teorías subjetivas sobre lo que es el conocimiento, la enseñanza y el aprendizaje.

La otra incoherencia que se produce se da al nivel de las respuestas, en donde tienen poca incidencia las innovaciones con teóricos e incluso empíricos soportes, al igual que ciertas estrategias fundamentales en el contexto de la innovación (JEFFERY, \& HALCOMB-SMITH, 2020). La innovación, que está definitivamente ligada al adecuado desarrollo del conocimiento y el aprendizaje, es un elemento fundamental de los nuevos modos de producción del conocimiento (BARAC, PRESTRIDGE, \& MAIN, 2017).

Los nuevos modos de producción del conocimiento están ejerciendo una presión sobre la universidad -en cuyo juego de presiones no aparecen como el único factor, también están otras instituciones y muy particularmente los gobiernos-, manteniéndola en una tensión de la que la propia universidad todavía no se ha mentalizado más que parcialmente. Los programas de Investigación, Desarrollo e Innovación, conocidos bajo las siglas I+D+i, son buena prueba de ello, en donde determinadas líneas responden más a cuestiones de modelo social, cultural o económico, es decir, a presiones ideológicas, que a verdaderas necesidades sociales. $\mathrm{O}$, si se quiere, la cuestión expresada al contrario, es decir, evidenciar la no existencia de protocolos de investigación, colaboración, o intervención basados en alianzas de conocimiento, que deja en mal lugar el abordaje de problemas sobrevenidos.

Ya dentro de las universidades, en el ámbito de la planificación del currículum, ¿se enfoca el desarrollo del mismo centrado en los procesos, o en los resultados? Se trata de una designación pertinente a lo que mantiene en distinción clara aquellos dos planteamientos didácticos, en donde de forma bipolar se le da importancia a "lo que está teniendo lugar" previo a los efectos o, en el otro extremo, "lo que ha de ocurrir", todo lo cual en una clara referencia a 
los procesos o a los resultados, los cuales se han de cuidar indistintamente.

Finalmente, otra incoherencia que se da ocurre a nivel organizativo. Esta se manifiesta en escenarios nacionales e internacionales. La universidad mantiene elementos (burocratización, masificación, inflexibilidad de normas, etc.) que han llegado a un punto donde antes que favorecer el libre desarrollo de los fines y funciones de la universidad, en relación al aprendizaje y la enseñanza, entorpecen o deterioran los procesos, de tal forma que se crea un claro perjuicio para quienes pasan por la universidad. Esta cuestión puede ser ejemplificada con dos ejemplos.

Por un lado, ciertos programas de intercambio, como el caso del Programa Europeo Erasmus, han sido establecidos sin resolver previamente las trabas burocráticas, académicas, curriculares, etc. que le afectaban (BAUTISTA-VALLEJO, ESPIGARES-PINAZO, \& HERNÁNDEZ-CARRERA, 2019). De esta forma, las experiencias de este alumnado de intercambio no han sido del todo satisfactorias, por lo menos por la parte académica.

Por otro lado, en el interior de las instituciones universitarias se maneja una noción de investigación y de innovación muy pobre, exhausta (BARNETT, \& BENGTSEN, 2017). Al alumnado se le exigen determinadas competencias en estas áreas, pero no existe realmente una formación transversal en las mismas que recorra los estudios, fundamentalmente de grado, de este alumnado. El resultado es la falta de competencia en estas áreas, lo que repercute negativamente en la capacidad profesional de los mismos.

Tradicionalmente, las tres manifestaciones aludidas evidencian una serie de signos visibles en la práctica (BARNETT, 2019).

En primer lugar, aparecen discrepancias en el "discurso" oficial universitario. El significado que puedan tener conceptos como trabajo, competencia y eficiencia aparecen mezclados con aquellos otros como concienciación, diálogo y responsabilidad ética. Por ejemplo, en torno a la responsabilidad cabe preguntarse: ¿qué papel juega la misma en relación a las tradicionales funciones universitarias de enseñanza, investigación y enseñanza de una profesión?

En segundo lugar, se presenta una ruptura en relación a las visiones del currículum universitario. El mismo podría verse como un conjunto de experiencias diseñadas a priori, las cuales son ofrecidas al alumnado, o como un conjunto de experiencias en donde el alumnado tiene un importante peso específico en su conformación. Por ejemplo, durante la reforma universitaria en Europa, el llamado proceso de Bolonia, en el origen de las discusiones sobre el nuevo instrumento de medida del trabajo del estudiante, el ECTS (crédito europeo), se estimaba que éste debía ser una herramienta para medir una variedad de aspectos, entre ellos los 
propuestos por la institución (el currículum), pero, también, aquellas otras actividades de formación propuestas por parte del alumnado. Todo sumaba en el cómputo ECTS, si bien esto último nunca se llevó a cabo.

Más allá de la política universitaria, las concepciones del currículum no son más que llevar a la práctica la visión de los actores universitarios, en un escenario donde tener poder es muy importante para imponer una determinada visión acerca de lo que significa crear, gestionar y transmitir el conocimiento, curricular o no, visible o invisible. Véase, por ejemplo en este sentido, el contraste entre la fetichización de la tecnología educativa y la falta de instrucción estructurada de alfabetización digital, lo cual puede constituir un currículum oculto con alguna intención no declarada (DARVIN, 2019; SILVA, 2011).

En tercer lugar, hay una serie de discrepancias que afectan a la filosofía subyacente. Es así que podría preguntarse: ¿qué objetivos educativos deberían ser trabajados en la universidad? La pregunta parece obvia, pero el modelo por competencias ha traído nuevos significados a la enseñanza y aprendizaje universitarios (BAUTISTA VALLEJO, \& LÓPEZ JARA, 2019) y la paradoja de la incoherencia en torno a las teorías adoptadas y teorías en uso en relación a la adopción del modelo por competencias.

La evidencia de estas últimas discrepancias plantea nuevas cuestiones. Por ejemplo, ¿qué significa que la universidad capacite profesionalmente? ¿Forma parte de ese conjunto de conocimientos la capacidad para resolver problemas de los que nunca fue hablado el alumnado? ¿Debe empeñarse la universidad en capacitar emprendedores que generen nuevas soluciones y, también, nuevos problemas al corpus de conocimientos? ¿Debe contribuir de una manera decisiva a la propia realización personal del estudiante?

En una institución que manifiesta todas estas incoherencias y discrepancias, el currículum y, dentro de él, la enseñanza y aprendizaje, genera un verdadero conflicto en relación a lo profesional y lo intelectual que, separados del mundo, devuelve de nuevo la pregunta de ¿cuáles son los objetivos de formación que deberían ser trabajados?

\section{Conclusiones}

Este trabajo explora, bajo la guía de una serie de categorías y a través de una espiral auto-reflexiva continua (HERNÁNDEZ CARRERA, 2014), las nuevas relaciones entre los miembros de la comunidad universitaria, en el contexto de una de las funciones de la universidad: la producción de conocimiento.

A lo largo de estas páginas se han vertido análisis de aspectos relevantes y la controversia que puede haber sobre la cuestión, en el marco de los significados que se otorga 
en la actualidad al conocimiento, con presiones importantes bajo la crítica de la obsolescencia del mismo, de las nuevas formas de producción de éste y la necesidad de innovación en el contexto de otra de las funciones universitarias: las formas que adquiere la enseñanza y el aprendizaje en las instituciones de educación superior y su vehículo el currículum.

Las transformaciones en todos estos terrenos se estaban dando desde hacía años, por lo menos desde mitad del siglo XX, tras la Segunda Guerra Mundial. Sin bien esto, la pandemia de este siglo, aquella provocada por el COVID-19, puede estar produciendo una aceleración en estos procesos como consecuencia del impacto, las nuevas dinámicas docentes y la tecnología, esta vez en un experimento de alcance mundial sin precedentes.

Algo nuevo que podría apuntarse en la universidad del siglo XXI, pero que conecta con la naturaleza de la misma, es que dentro de ella sea posible entender una constelación de la producción (científica, cultural, personal, etc.) y el valor de todo ello para una nueva sociedad cognitiva que se crea, ahora más rápidamente, por el impacto de la tecnología. Al igual que esta primera, también podría hablarse de una constelación de la democracia y del valor asignado a la misma, una constelación de la emancipación, a la que debe conducir o no la formación universitaria, y, finalmente, una constelación del sujeto, de la persona, cuando nos preguntamos si ésta no está a la base o por encima de todas las demás.

En este sentido, lo esencial de las competencias en los nuevos currículums debería consistir en la comprensión. Esta es la manera en la que los estudiantes aprehenden y disciernen fenómenos relacionados con una asignatura, antes bien que lo que los estudiantes conocen acerca de estos fenómenos o cómo los manipulan. Es así que muchos estudiantes pueden reproducir el conocimiento memorizado de un libro de texto, mientras no están comprendiendo sus asignaturas.

\section{REFERENCIAS}

ADAMS, S., CUMMINS, M., DAVIS, A., FREEMAN, A., HALL, C. Y ANANTHANARAYANAN, V. NMC Horizon Report: 2017 Higher Education Edition. Austin, Texas: The New Media Consortium. 2017.

ALFONSO SÁNCHEZ, I. R. La Sociedad de la Información, Sociedad del Conocimiento y Sociedad del Aprendizaje. Referentes en torno a su formación. Bibliotecas. Anales de investigación, 12(2), 235-243. 2016.

BARAC, K., PRESTRIDGE, S., \& MAIN, K. Stalled Innovation: Examining the technological, pedagogical and content knowledge of Australian university educators. Australian Educational Computing, 32(1), 1-17. 2017. Recuperado de: http://hdl.handle.net/10072/348562 
BARDIN, L. Análisis de contenido. Madrid: Akal. 1996.

BARNETT, R. Towards a higher education for a new century. London: Institute of Education, University of London. 1997.

BARNETT, R. Learning for an unknown future. Higher Education Research \& Development, 23(3), 247-260. 2004.

BARNETT, R. The Thoughtful University: A Feasible Utopia. Beijing International Review of Education, 1(1), 55-72. 2019. https://doi.org/10.1163/25902547-00101007

BARNETT, R., \& BENGTSEN, S. Universities and Epistemology: From a Dissolution of Knowledge to the Emergence of a New Thinking. Education Sciences, 7(1), 38. 2017. https://doi.org/10.3390/educsci7010038.

BARNETT, R., \& BENGTSEN, S. S. Knowledge and the university: Re-claiming life. New York: Routledge. 2019.

BAUTISTA VALLEJO, J. M. Universidad y Espacio Europeo en la encrucijada de la calidad. Huelva: Hergué. 2005.

BAUTISTA VALLEJO, J. M., \& LÓPEZ JARA, N. R. Critical analysis of the competencebased model in the University. Academo (Asunción), 6(1), 71-80. 2019.

BAUTISTA-VALLEJO, J. M., ESPIGARES-PINAZO, M. J., \& HERNÁNDEZ-CARRERA, R. M. El eees en la Universidad española como innovación y el papel del alumnado veinte años después. En: F. J. Hinojo-Lucena, I. Aznar-Díaz, \& Ma . P. Cáceres Reche (eds), Avances en recursos TIC e innovación educativa (pp. 139-151). Madrid: Dykinson. 2019.

http://dx.doi.org/10.2307/j.ctv105bcf5.15.

BECHER, T. Academic Tribes and Territories. Buckingham: Open University Press. 1989.

BONARDI, J. P., BRIS, A., BRÜLHART, M., DANTHINE, J. P., JONDEAU, E., ROHNER, D., THOENIG, M. The Case for Reopening Economies by Sector. Harvard Business Review, May. 2020. Recuperado de https://hbr.org/2020/05/the-case-for-reopening-economies-bysector.

BOUZA MORA, D. Instrucción: UNA-VD-DISC-003-2020. Modificación de las actividades académicas durante la situación de emergencia por COVID19 en la Universidad Nacional. San José, Costa Rica: Universidad Nacional. 2020.

BROWN, J., BROCK, B., \& ZÁVODSKÁ, A. Higher Education in the 21st century: A New Paradigm of Teaching, Learning and Credit Acquisition. Proceedings of The Multidisciplinary Academic Conference, 14th IAC 2019, 87-94. 2019.

BRUNNER, J. J. Transformación de lo público y el reto de la innovación universitaria. Bordón. Revista de Pedagogía, 66(1), 45-60. 2014.

BURKE, P. A social history of knowledge: from Gutenberg to Diderot. Cambridge, UK: Polity Press. 2015. 
COMISIÓN DE LAS COMUNIDADES EUROPEAS. Por una Europa del conocimiento. Comunicación al Consejo, al Parlamento Europeo, al Comité Económico y Social y al Comité de las Regiones. Bruselas, 12.11.1997. COM (97) 563 final. 1997.

COMMITTEE OF SCOTTISH UNIVERSITY PRINCIPALS. Teaching and Learning in an Expanding Higher Education System. Edinburgh: SCFC. 1991.

DARVIN, R. Youth, Technology, and the Hidden Curriculum of the 21st Century. Youth and Globalization, 1(2), 210-229. 2019.

DE KRUMMEL, M. D., BAUTISTA-VALLEJO, J. M., HERNÁNDEZ, R., \& ESPIGARESPINAZO, M. J. La autonomía universitaria como problema educativo: Una orientación histórica. ACADEMO Revista de Investigación en Ciencias Sociales y Humanidades, 7(1), 8996. 2020.

DZIB GOODIN, A.; CASTEVICH, J.; HOGAN, D.L.; SANDERS, L.; SLOVEC, K. Y YELIZAROV, D. Desarrollo del entorno personal de aprendizaje para tutoría e investigación en niveles educativos superiores. Innoeduca. International Journal of Technology and Educational Innovation, 1(1), junio, 10-16. 2015. doi:

http://dx.doi.org/10.20548/innoeduca.2015.v1i1.27

FINN, M. The Never-Ending Crisis in British Higher Education. En: T. Geelan, M. González Hernando, \& P. William Walsh (eds), From Financial Crisis to Social Change (pp. 33-51). Cham: Palgrave Macmillan. 2018.

GARCÍA-PEÑALVO, F. J. La tercera misión. Education in the Knowledge Society, 17(1), 718. 2016.

GEORGE, J. F., \& MARETT, K. Invited Paper: The Times they are a Changin: How NonTechnology Factors have Affected IS Curriculum over Time. Journal of Information Systems Education, 30(4). 2019. Recuperado de https://aisel.aisnet.org/jise/vol30/iss4/3.

GIBBONS, M., LIMOGES, C., NOWOTNY, H., \& SCHWARTZMAN, S. La nueva producción del conocimiento. La dinámica de la ciencia y la investigación en las sociedades contemporáneas. Barcelona: Pomares-Corredor. 1997.

GUZMÁN-VALENZUELA, C. Universities, knowledge and pedagogical configurations: Glimpsing the complex university. Educational Philosophy and Theory, 50(1), 5-17. 2017. http://dx.doi.org/10.1080/00131857.2017.1313717

HERNÁNDEZ CARRERA, R. M. La investigación cualitativa a través de entrevistas: su análisis mediante la teoría fundamentada. Cuestiones Pedagógicas, 23, 187-210. 2014.

ICART ISERN M. T. Y CANELA-SOLER, J. El artículo de revisión. Enfermería Clínica, 4(4), 180-184. 1994.

JEFFERY, K., \& HALCOMB-SMITH, L. Innovation, Critical Pedagogy, and Appreciative Feedback: A Model for Practitioners. En: S. Palahicky (2020), Enhancing Learning Design for Innovative Teaching in Higher Education (pp. 1-21). Hershey, Pennsylvania, USAIGI Global. 2020. http://dx.doi.org/10.4018/978-1-7998-2943-0.ch001 
KAUARK, F. D. S., MANHÃES, F. C. Y MEDEIROS, C. H. Metodologia da pesquisa: um guía prático. Itabuna: Via Litterarum. 2010.

KNOWLEDGE EXCHANGE CONCORDAT. Concordat for the advancement of knowledge exchange in higher education. Universities UK. 2020.

LYOTARD, J. F. La condición postmoderna. Madrid: Cátedra. 1994.

MATOS-DE-SOUZA, Rodrigo. Quand la politique se manifeste dans un contexte de crise pandémique. In . Breton, Hervé. Chronique du vecu d'une pandemie planetaire: Récits d'universitaires, d'Est en Ouest, premier semestre 2020, Paris: L'Harmattan, p. 195-201.

MUÑOZ, J. L. Y LLUCH, L. Educación y COVID-19: Colaboración de las familias y tareas escolares. Revista Internacional de Educación para la Justicia Social, 9(3e). 2020.

REAL DECRETO-LEY 8/2020, de 17 de marzo, de medidas urgentes extraordinarias para hacer frente al impacto económico y social del COVID-19. 2020.

SCOTT, P. The Meanings of Mass Higher Education. Buckingham: Open University Press. 1995.

SILVA, A. M. A Uberização do Trabalho Docente no Brasil: uma tendência de precarização No Século XXI. Trabalho Necessário, 17(34), 230-251. 2019.

SILVA, T. T. Documento de Identidade: uma introdução às teorias do currículo. Belo Horizonte: Autêntica. 2011.

SKIBA, D. J. Horizon Report: Knowledge Obsolescence, Artificial Intelligence, and Rethinking the Educator Role. Nursing Education Perspectives, 38(3), 165-167. 2017.

SOHRABI, C., ALSAFI, Z., O’NEILL, N., KHAN, M., KERWAN, A., AL-JABIR, A., IOSIFIDIS, C., \& AGHA, R.World Health Organization declares global emergency: A review of the 2019 novel coronavirus (COVID-19). International Journal of Surgery, 76, April, 7176. 2020. https://doi.org/10.1016/j.ijsu.2020.02.034

STANDING, G. O precariado: a nova classe perigosa. Belo Horizonte: Autêntica. 2017.

SUND, K. J. Suggestions for Moving Teaching Rapidly Online in the Face of the Corona Crisis. Roskilde: Roskilde University. 2020.

VENCO, S. Uberização do trabalho: um fenômeno de tipo novo entre os docentes de São Paulo, Brasil? Cadernos de Saúde Pública, 35. 2019. (Suppl 1). https://doi.org/10.1590/0102$311 \mathrm{X} 00207317$

WANG, C., CHENG, Z., YUE, X. G., \& MCALEER, M. Risk Management of COVID-19 by Universities in China. Journal of Risk and Financial Management, 13(2), 1-6. 2020. https://doi.org/10.3390/jrfm13020036

WANG, G., ZHANG, Y., ZHAO, J., ZHANG, J., \& JIANG, F. Mitigate the effects of home confinement on children during the COVID-19 outbreak. The Lancet, March 4th, 1-2. 2020. https://doi.org/10.1016/S0140-6736(20)30547-X 
WHEELAHAN, L. What kind of curriculum, pedagogy and qualifications do we need for an uncertain future? En: Conference Proceedings of 'What a Difference a Pedagogy Makes: Researching Lifelong Learning and Teaching', 2, 635-640. Stirling: CRLL. 2005.

\section{SOBRE LOS AUTORES:}

\section{José M. Bautista-Vallejo}

Doctor europeo en Psicopedagogía por la Universidad de Huelva. Premio Extraordinario de Doctorado. Doctor Honoris Causa por la Universidad Iberoamericana (Paraguay). Profesor Titular en la Universidad de Huelva. Departamento de Pedagogía de la Universidad de Huelva. E-mail: bautista@uhu.es

(iD https://orcid.org/0000-0002-4238-9507

\section{Rafael M. Hernández-Carrera}

Doctor europeo en educación por la Universidad de Sevilla, Profesor y Director del Departamento de Didáctica y Organización Escolar de la Universidad Internacional de la Rioja. Docente del Master en Liderazgo y Dirección de Centros Educativos de la Universidad Internacional de la Rioja. E-mail: rafael.hernandez@unir.net

(iD https://orcid.org/0000-0002-7722-8105

\section{Rodrigo Matos-de-Souza}

Doutor em Educação e Contemporaneidade pela Universidade do Estado da Bahia - UNEB. Professor da Universidade de Brasília, Brasil. Docente do Programa de Pós-Graduação em Educação - Modalidade Profissional. Líder do Grupo de Pesquisa Rede Experiência, Narrativas e Pedagogias da Resistência (REDExp). E-mail: rodrigomatos@unb.br (iD https://orcid.org/0000-0002-8788-4966 